\title{
Industrial Integration in Changchun and Jilin City Based on the Similar Coefficients of Industrial Structure
}

\author{
Huimin Sun, Jinling Wang
}

College of Economy, Changchun University, Changchun, China.

Email:shm1982@ sina.com

Received November $30^{\text {th }}$, 2012; revised December 31 ${ }^{\text {st }}$, 2012; accepted January $20^{\text {th }}, 2013$

Copyright (c) 2013 Huimin Sun, Jinling Wang. This is an open access article distributed under the Creative Commons Attribution License, which permits unrestricted use, distribution, and reproduction in any medium, provided the original work is properly cited.

\begin{abstract}
This paper primarily calculates the similar coefficients of the industrial structure in Changchun and Jilin City, the results show that the similarity of the three industries in the two cities is high, but the similarity of the subdivided manufacture industries is low. We also find evidence that the conditions for the industrial integration of the two cities are improved. The paper also explores the reasons for the current situation of the industrial integration in Changchun and Jilin city, then propose the measures to develop the industrial integration.
\end{abstract}

Keywords: Changchun and Jilin City; Similarity Coefficient of Industrial Structure; Industrial Integration

\section{Introduction}

The distance between Changchun and Jilin city is less than 90 kilometers, the population in the two cities accounts for $45 \%$ of that in Jilin province, the GDP in the two cities accounts for more than $60 \%$ and the local financial revenue is above $40 \%$ of that in Jilin province. Changchun and Jilin city are also the central areas of Changchun-Jilin-Tumen forerunner zone. As to the industrial development, the pillar industries of Jilin province such as automobile industry, chemical industry and agricultural products processing industry are all located in the two cities. The industrial integration in Changchun and Jilin city (written as Changchun-Jilin in the following text) means being grounded on the pillar industries in the two cities, the reasonable allocation of factors should be realized and the local economy growth should depend on the horizontal and vertical division of labor and cooperation, and on the establishment of common market and industrial convergence.

Under the background of economic globalization, when one country or region is involved in the international division of labor, it no longer depends on the power of one single city or one province, but increasingly depends on several cities or regions. The economy in these cities or regions should be developed simultaneously by means of resource integration, cooperation and division of labor.

The paper analyzes the development situation of the industrial integration in Changchun-Jilin by use of the Similar Coefficients of Industrial Structure.

\section{Previous Research}

The existing domestic research on the industrial integration focuses on that in Yangtze River delta and BeijingTianjin-Hebei district, only few papers research the industrial integration in Changchun-Jilin. H. Wang and Y. P. Wang (2011) [1] studied the problems in the process of industrial integration in Changchun-Jilin. Their study suggested that the industrial relevancy in the region was low, the industrial structure was similar but the technique relevancy on the industry chains was little. These problems have restricted the development of the industrial integration. The study of R. Q. Pang and J. Li (2011) [2] showed that in the north-east of China, because the market participants were not active and lacked enterprise consciousness, the development of industrial integration was restricted. W. Li and H. J. Liu (2012) [3] found that the problem in the process of industrial integration in Changchun-Jilin was that the projects under cooperation were mainly infrastructure, real estate, tourism and public service. Industries with great relevancy and long industry chains and high-tech industries were few. Their findings suggested that the industries in the integration process were at low level.

In the research of the relationship between the similarity of industrial structure and the level of industrial inte- 
gration, W. Li and W. H. Xia (2011) [4] studied the Industrial integration of Wuhan Urban Agglomeration, they concluded that the high coefficient of industrial structure will affect the overall advantage and the general benefit in the region. But some scholars presented different opinions. For example, J. J. Chen (2004) [5] concluded in his paper that the similarity of industrial structure is related to the similarity of resource endowment; the major problem in the industrial integration in Yangtze River delta is not repeated construction, but the waste of resource caused by vicious competition and the disordering of the market regulation.

In the studies about the effect of local industrial integration on the local economy, the study of X. Li and J. B. Yin (2012) [6] showed that regional industrial integration could enlarge the market, promote competition and lead to industrial cluster and regional specialization. In the research about the approach to develop regional Industrial integration, L. M. Zhao and L. Zhang (2011) [7] proposed to adjust the industrial distribution across different regions, the local governments need to establish communication and negotiation system and to optimize the factor allocation in a larger market by means of sound systems and policies.

\section{Emprical Analysis of the Industrial Structure Similarity in Changchun-Jilin}

\subsection{Analysis of the Three-Industry-Structure Similarity in Changchun-Jilin}

Many methods can be used in the calculation of the similarity of regional industrial structure, in which a commonly used one is the similar coefficient of industrial structure presented by the United Nations Industrial Development Organization (UNIDO) in 1979. This method is used in the following text to calculate the similarity degree of industrial structure in Changchun-Jilin. The expression of the similarity of regional Industrial structure is:

$$
S i j=\sum_{k=1}^{n} X i k \times X j k / \sqrt{\sum_{k=1}^{n} X i k^{2} \times \sum_{k=1}^{n} X j k^{2}}
$$

where $S i j$ refers to the similarity coefficient of Industrial structure of the two regions $i$ and $j, X i k$ represents the proportion of industry $k$ in the general output value(here the total industrial output is selected) in region $i, X j k$ represents the proportion of the Industry $k$ in the general output value (here the total industrial output is selected) in region $j$. The value of $S i j$ is between 0 and 1 . If $S i j=0$, it means that the industrial structure in regions $i$ and $j$ is totally different, if $S i j=1$, it means that the industrial structure in regions $i$ and $j$ is totally the same. The larger the value of Sij, the more similar the industrial structure of the two regions is.

The above mentioned method has been extensively used by many scholars and experts. W. S. Wang (2007) used this method to calculate the similar coefficient of industrial structure of Guangzhou and Shenzhen, and further analyzed the industrial structure of Guangdong province [8]. J. J. Zhuo, S. H. Wei and X. J. Liu (2011) adopted the method to research the industrial integration in Yangtze River Delta Area, found that the similarity of manufacturing industry between Jiangsu and Shanghai was increasing, while the similarity of manufacturing industry between Jiangsu and Zhejiang was decreasing [9]. Y. W. and J. Q. X. (2011) Used the method to analyze the developing trend of industrial structure in Shenzhen and the result showed that the industrial structure in Shenzhen is increasingly similar to that in Pearl River Delta [10].

The total output of the three industries and the value of GDP in Changchun and Jilin city are selected and the similar coefficients of the three industries from the year 2005 to 2011 are calculated. Table 1 shows the proportion of the three industries in the local GDP and the similar coefficients of industrial structure in ChangchunJilin from the year 2005 to 2011.

The results of Table 1 show that the similarity of the three industries in Changchun-Jilin is rather high. The three-industrial-structure is changing with the economic development. According to the industrial structure theory, Jilin province is in the middle term of industrialization. In the three industries, the proportion of the primary industry is low, the proportion of the secondary industry is increasing year by year, and the proportion of the tertiary industry is increasing obviously, which show the characteristics in the middle term of industrialization. But the proportion of the primary industry in Changchun is lower than that in Jilin city, and the proportion of the tertiary industry in Changchun is higher, which suggests that the three-industry-structure in Changchun is more advanced. In the process of industrialization, the proportion of the secondary industry will decrease, but the proportion of the tertiary industry will increase and further become dominate. In the year of 2011, the proportion of the primary, the secondary and the tertiary industry in China is $10.1 \%, 46.8 \%$, and $43.1 \%$ respectively. We can conclude that the development of the tertiary industry in Changchun and Jilin city falls behind other cities and provinces all over the country. The three-industry-structure should be further adjusted in order to push the industrial integration in Changchun-Jilin.

\subsection{Similarity Analysis of the Manufacture Industry in Changchun-Jilin}

The secondary industry has taken the largest part of GDP in Changchun and Jilin city, and manufacture industry is 
Table 1. Proportion of three industries in local GDP and the similar coefficients of industrial structure in Changchun-Jilin.

\begin{tabular}{|c|c|c|c|c|c|c|c|}
\hline \multirow{2}{*}{ Year } & \multicolumn{3}{|c|}{ Changchun city } & \multicolumn{3}{|c|}{ Jilin city } & \multirow{2}{*}{$\begin{array}{c}\text { Similar coefficien } \\
\text { of the three } \\
\text { industries }\end{array}$} \\
\hline & Primary industry & Secondary industry & Tertiary industry & Primary industry & Secondary industry & Tertiary industry & \\
\hline 2005 & 0.107 & 0.470 & 0.423 & 0.160 & 0.450 & 0.391 & 0.9954 \\
\hline 2006 & 0.093 & 0.489 & 0.418 & 0.152 & 0.457 & 0.392 & 0.9947 \\
\hline 2007 & 0.096 & 0.502 & 0.402 & 0.131 & 0.496 & 0.373 & 0.9978 \\
\hline 2008 & 0.085 & 0.512 & 0.403 & 0.129 & 0.489 & 0.382 & 0.9972 \\
\hline 2009 & 0.079 & 0.506 & 0.415 & 0.114 & 0.493 & 0.393 & 0.9982 \\
\hline 2010 & 0.076 & 0.517 & 0.407 & 0.108 & 0.498 & 0.394 & 0.9986 \\
\hline 2011 & 0.072 & 0.523 & 0.405 & 0.100 & 0.505 & 0.395 & 0.9990 \\
\hline
\end{tabular}

the important one in the secondary industry. Thus, the similarity of the manufacture industries in ChangchunJilin is analyzed here.

According to the availability of data, the total output of the industrial enterprises above designated size is selected and the total output of 27 subdivided manufacture industries are analyzed. After calculation, the similarity coefficients of the manufacture industry structure in Changchun-Jilin are shown in Table 2 from the year 2005 to 2009.

The results of Table 2 show that the similarity of the manufacture industry structure in Changchun-Jilin is very low in spite of the high similarity of the three-industry-structure. This is because the emphasis of the manufacture industries in Changchun and Jilin city is different. The transportation equipment manufacturing is the leading industry in Changchun, the output of which accounted for $65.2 \%, 73.1 \%, 71.8 \%, 73.1 \%, 59.4 \%$ of the total industrial output respectively in Changchun from the year 2005 to 2009. While the leading industry in Jilin city is manufacture of raw chemical materials and chemical products, the output of which accounted for $57.3 \%$, $55.2 \%, 46.9 \%, 38.0 \%$ and $33.7 \%$ of the total industrial output respectively in Jilin city from the year 2005 to 2009. The correlativity between the two leading Industries in the two cities is low and there are few main industries that can be jointly developed. Thus, great difficulty still exists in the industrial integration in Changchun-Jilin.

Except in the year of 2006, the similarity coefficients of the manufacture industries in Changchun-Jilin were growing year by year from 2005 to 2009. This shows that the industry correlativity in Changchun-Jilin is improving and the conditions for industrial integration are perfected. For instance, the gap between the proportion of processing of food from agricultural products (another advantage industry in both cities) was narrowed, from $9.5 \%$ in 2005 to $3.2 \%$ in 2009 .

\section{Measures to Develop Industrial Integration in Changchun-Jilin}

First, to promote the integral development of the leading sectors in Changchun-Jilin. The forerunner of development economics Walt Whitman Rostow presented that leading industries can promote local economic growth, can decide and change local industrial structure [11]. He called the effect of leading sectors on local economic growth as spreading effect. The value of the location quotient of the transportation equipment manufacturing in Changchun was 8.5 in 2009, and the value of the location quotient of the manufacture of raw chemical materials and chemical products in Jilin city was 5 in the same year (calculated based on the data from China Statistical Yearbook 2010, Changchun Statistical Yearbook 2010 and Jilin City Economic and social statistical year-book 2010). So we conclude that the transportation equipment manufacturing and the manufacture of raw chemical materials and chemical products are the leading industries of Changchun and Jilin city respectively. Both industries have long industrial chains, and the chains of propylene oxide, synthetic rubber and synthetic resin on the manufacture industry of raw chemical materials and chemical products can be connected with automobile industry. Other products, such as metal manufacturing, metal smelting and calendering industry of Jilin city are correlated with the automobile industry of Changchun and can be jointly developed. Simultaneously, the development of leading industry can bring the growth of correlated industries. For example, the development of automobile industry will lead to the growth of automobile culture, tourism and logistics industry.

Second, to establish industrial parks. Because of the concentration of enterprises in the industrial parks, the cost of information transmission can be reduced and the factor mobility can be accelerated. Meanwhile, the sharing of resource, equipment and information in the Industrial parks can lead to technology diffusion. Enterprises 
Table 2. Similarity coefficients of manufacture industry structure in Changchun-Jilin.

\begin{tabular}{cccccc}
\hline year & 2005 & 2006 & 2007 & 2008 & 2009 \\
$\begin{array}{c}\text { Similarity } \\
\text { coefficients }\end{array}$ & 0.0853 & 0.0836 & 0.0872 & 0.150 & 0.192 \\
\hline
\end{tabular}

in the industrial parks have stronger competition awareness, which can promote technology innovation and joint development of different enterprises and industries. The establishment of industrial parks in Changchun-Jilin can strengthen the industrial correlation in the two cities and create sound conditions for the industrial integration.

Finally, to work out overall developing strategies and to break administrative segmentation of local governments. In the process of industrial integration across different regions, the administrative segmentation is a common restrictive factor. Thus, the local governments of Changchun and Jilin city should clarify the developing goals and unify thoughts, establish industries connection system and service system, set up special organization for information communication, and avoid repeated construction and resource waste.

\section{Limitations and Future Research}

Based on data limitations we only calculate the similarity coefficients of manufacturing industry using the values of total output of the 27 subdivided industries. Further study should be able to calculate the similarity coefficients using the industrial added values.

\section{Acknowledgements}

The authors would like to acknowledge to staff form Approaches to Develop Industrial Integration of Changchun and Jilin City. This study was funded by the Changchun Municipal Science and Technology Bureau (Grant No. 11RY37).

\section{REFERENCES}

[1] H. Wang and Y. P. Wang, "Research on the Industrial Integration of Changchun and Jilin City,” Market Modernization, No. 1, 2011.

[2] R. Q. Pang and J. Li, "Developmental Strategies of Changchun-Jilin-Tumenjiang of Regional Industry under the Background of Integration,” Journal of Northeast Normal University (Philosophy and Social Sciences), No. 4, 2011.

[3] W. Li and H. J. Liu, "Countermeasures to Promote the Industrial Integration of Changchun and Jilin City,” Journal of the Party School of CPC Changchun Municipal Committee, No. 3, 2012.

[4] W. Li and W. H. Xia, "The SWOT Model Analysis of Industrial Integration of Wuhan Metropolis,” Future and Development, No. 7, 2011, pp. 108-112.

[5] J. J. Chen, "Study on Same Structure and Position of Industry in Delta Area of Changjiang River," China Industrial Economy, No. 2, 2004.

[6] X. Li and J. B. Yin, "Research Review on the Effects of Regional Integration on Industry," City Planning Review, No. 5, 2012.

[7] L. M. Zhao and L. Zhang, “The Base of Driving Power for Industrial Integration in the Area of Beijing, Tianjin and Hebei Province," Journal of Tianjin Normal University (Social Science), No. 6, 2011, pp. 11-16.

[8] W. S. Wang, "The Application of the Similar Coefficients of Industrial Structure in Statistical Analysis," China Statistics, No. 10, 2007.

[9] J. J. Zhuo, S. H. Wei and X. J. Liu, “The Industrial Integration of the Yangtze River Delta Area against the Background of the Similar Industrial Structure,” Modern Urban Research, 2011.

[10] Y. W. and J. Q. X., “Analysis of Shenzhen Industrial Structure Based on Similar Coefficients and VAR Model," Statistics and Decision, No. 21, 2011.

[11] G. P. Yang, "Industrial Economics,” Fudan University Press, Shanghai, 2005. 